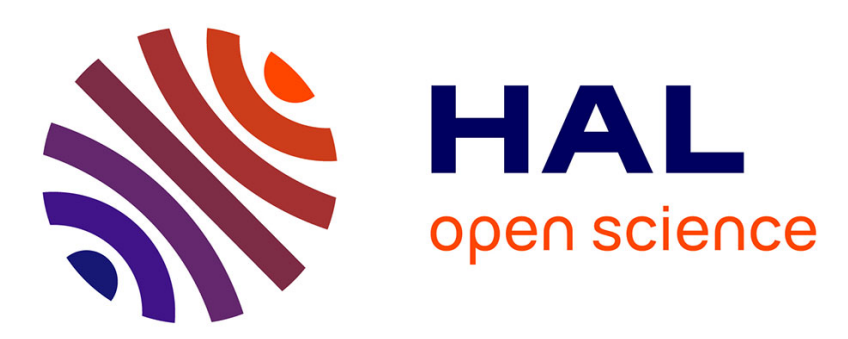

\title{
Existence of stationary solutions for some non-Fredholm integro-differential equations with superdiffusion
}

\author{
Vitali Vougalter, Vitaly Volpert
}

\section{To cite this version:}

Vitali Vougalter, Vitaly Volpert. Existence of stationary solutions for some non-Fredholm integrodifferential equations with superdiffusion. Journal of Pseudo-Differential Operators and Applications, 2016, 10.1007/s11868-016-0173-9 . hal-01397555

\section{HAL Id: hal-01397555 \\ https://inria.hal.science/hal-01397555}

Submitted on 16 Nov 2016

HAL is a multi-disciplinary open access archive for the deposit and dissemination of scientific research documents, whether they are published or not. The documents may come from teaching and research institutions in France or abroad, or from public or private research centers.
L'archive ouverte pluridisciplinaire HAL, est destinée au dépôt et à la diffusion de documents scientifiques de niveau recherche, publiés ou non, émanant des établissements d'enseignement et de recherche français ou étrangers, des laboratoires publics ou privés. 


\title{
Existence of stationary solutions for some non-Fredholm integro-differential equations with superdiffusion
}

\author{
Vitali Vougalter ${ }^{1}$, Vitaly Volpert ${ }^{2,3}$, \\ ${ }^{1}$ Department of Mathematics, University of Toronto \\ Toronto, Ontario, M5S 2E4, Canada \\ e-mail: vitali@math.toronto.edu \\ ${ }^{2}$ Institute Camille Jordan, UMR 5208 CNRS, University Lyon 1, Villeurbanne, 69622 France \\ ${ }^{3}$ Laboratoire Poncelet, UMI 2615 CNRS, Bolshoy Vlasyevskiy Pereulok 11 \\ 119002 Moscow, Russia \\ e-mail: volpert@math.univ-lyon1.fr
}

\begin{abstract}
We prove the existence of stationary solutions for some reaction-diffusion equations with superdiffusion. The corresponding elliptic problem contains the operators with or without Fredholm property. The fixed point technique in appropriate $H^{2}$ spaces is employed.
\end{abstract}

Keywords: solvability conditions, non Fredholm operators, integro-differential equations, stationary solutions, superdiffusion

AMS subject classification: 35J10, 35P30, 35K57

\section{Introduction}

We recall that a linear operator $L$ acting from a Banach space $E$ into another Banach space $F$ possesses the Fredholm property if its image is closed, the dimension of its kernel and the codimension of its image are finite. Consequently, the equation $L u=f$ is solvable if and only if $\phi_{i}(f)=0$ for a finite number of functionals $\phi_{i}$ from the dual space $F^{*}$. These properties of Fredholm operators are broadly used in many methods of linear and nonlinear analysis.

Elliptic problems in bounded domains with a sufficiently smooth boundary satisfy the Fredholm property if the ellipticity condition, proper ellipticity and Lopatinskii conditions are satisfied (see e.g. [1], [10], [16]). This is the main result of the theory of linear elliptic problems. When the domains are unbounded, these conditions may not be sufficient and the Fredholm property may not be satisfied. For instance, the Laplace operator, $L u=\Delta u$, in

$\mathbb{R}^{d}$ fails to satisfy the Fredholm property when considered in Hölder spaces, $L: C^{2+\alpha}\left(\mathbb{R}^{d}\right) \rightarrow$ $C^{\alpha}\left(\mathbb{R}^{d}\right)$, or in Sobolev spaces, $L: H^{2}\left(\mathbb{R}^{d}\right) \rightarrow L^{2}\left(\mathbb{R}^{d}\right)$. 
Linear elliptic equations in unbounded domains possess the Fredholm property if and only if, in addition to the conditions mentioned above, limiting operators are invertible (see [17]). In some trivial situations, limiting operators can be explicitly constructed. For instance, if

$$
L u=a(x) u^{\prime \prime}+b(x) u^{\prime}+c(x) u, \quad x \in \mathbb{R},
$$

where the coefficients of the operator have limits at infinity,

$$
a_{ \pm}=\lim _{x \rightarrow \pm \infty} a(x), \quad b_{ \pm}=\lim _{x \rightarrow \pm \infty} b(x), \quad c_{ \pm}=\lim _{x \rightarrow \pm \infty} c(x),
$$

the limiting operators are:

$$
L_{ \pm} u=a_{ \pm} u^{\prime \prime}+b_{ \pm} u^{\prime}+c_{ \pm} u .
$$

Since the coefficients are constant, the essential spectrum of the operator, that is the set of complex numbers $\lambda$ for which the operator $L-\lambda$ fails to satisfy the Fredholm property, can be explicitly found by means of the Fourier transform:

$$
\lambda_{ \pm}(\xi)=-a_{ \pm} \xi^{2}+b_{ \pm} i \xi+c_{ \pm}, \quad \xi \in \mathbb{R} .
$$

Invertibility of limiting operators is equivalent to the condition that the origin does not belong to the essential spectrum.

In the case of general elliptic equations, the same assertions are true. The Fredholm property is satisfied if the essential spectrum does not contain the origin or if the limiting operators are invertible. However, these conditions may not be explicitly written.

In the case of non-Fredholm operators the usual solvability conditions may not be applicable and solvability conditions are, in general unknown. There are some classes of operators for which solvability conditions are derived. We illustrate them with the following example. Consider the problem

$$
L u \equiv \Delta u+a u=f
$$

in $\mathbb{R}^{d}$, where $a$ is a positive constant. The operator $L$ coincides with its limiting operators. The homogeneous problem has a nonzero bounded solution. Thus the Fredholm property is not satisfied. However, since the operator has constant coefficients, we can use the Fourier transform and find the solution explicitly. Solvability conditions can be formulated as follows. If $f \in L^{2}\left(\mathbb{R}^{d}\right)$ and $x f \in L^{1}\left(\mathbb{R}^{d}\right)$, then there exists a unique solution of this equation in $H^{2}\left(\mathbb{R}^{d}\right)$ if and only if

$$
\left(f(x), \frac{e^{i p x}}{(2 \pi)^{\frac{d}{2}}}\right)_{L^{2}\left(\mathbb{R}^{d}\right)}=0, \quad p \in S_{\sqrt{a}}^{d} \quad \text { a.e. }
$$

(see [25]). Here and further down $S_{r}^{d}$ denotes the sphere in $\mathbb{R}^{d}$ of radius $r$ centered at the origin. Thus, though the operator fails to satisfy the Fredholm property, solvability conditions are formulated similarly. However, this similarity is only formal since the range of the operator is not closed. 
In the case of the operator with a potential,

$$
L u \equiv \Delta u+a(x) u=f,
$$

Fourier transform is not directly applicable. Nevertheless, solvability conditions in $\mathbb{R}^{3}$ can be obtained by a rather sophisticated application of the theory of self-adjoint operators (see [19]). As before, solvability conditions are formulated in terms of orthogonality to solutions of the homogeneous adjoint equation. There are several other examples of linear elliptic operators without Fredholm property for which solvability conditions can be derived (see [17]-[25]).

Solvability conditions play are crucial for the analysis of nonlinear elliptic equations. In the case of operators without Fredholm property, in spite of some progress in understanding of linear equations, there exist only few examples where nonlinear non-Fredholm operators are analyzed (see [5]-[7]). In the present article we study another class of nonlinear equations, for which the Fredholm property may not be satisfied:

$$
\frac{\partial u}{\partial t}=-\sqrt{-\Delta} u+a u+\int_{\Omega} G(x-y) F(u(y, t), y) d y, \quad a \geq 0 .
$$

Here $\Omega$ is a domain in $\mathbb{R}^{d}, \quad d=1,2,3$, the more physically interesting dimensions. The operator $\sqrt{-\Delta}$ here is defined by means of the spectral calculus. Model (1.2) describes a particular case of superdiffusion actively studied in the context of different applications in plasma physics and turbulence (see e.g. [4], [15]), surface diffusion (see e.g. [11], [13]), semiconductors (see e.g. [14]) and so on. The superdiffusion can be understood as a random process of particle motion characterized by the probability density distribution of jump length. The moments of this density distribution are finite in the case of normal diffusion, but this is not the case for superdiffusion. Asymptotic behavior at infinity of the probability density function determines the value of the power of the negative Laplacian (see e.g. [12]).

In population dynamics the integro-differential equations describe models with intraspecific competition and nonlocal consumption of resources (see e.g. [2], [3], [8]). We will use the explicit form of solvability conditions and will study the existence of stationary solutions of the nonlinear problem.

When $a$ is not a constant, we expect the stationary solutions of (1.2) to exist under the appropriate technical conditions. However, the method of the proof of the present article heavily relies on the Fourier transforms and therefore, it cannot be applied directly when $a$ depends on $x$.

\section{Formulation of the results}

The nonlinear part of problem (1.2) will satisfy the following regularity conditions.

Assumption 1. Function $F(u, x): \mathbb{R} \times \Omega \rightarrow \mathbb{R}$ is such that

$$
|F(u, x)| \leq k|u|+h(x) \quad \text { for } \quad u \in \mathbb{R}, x \in \Omega
$$


with a constant $k>0$ and $h(x): \Omega \rightarrow \mathbb{R}^{+}, \quad h(x) \in L^{2}(\Omega)$. Moreover, it is a Lipschitz continuous function, such that

$$
\left|F\left(u_{1}, x\right)-F\left(u_{2}, x\right)\right| \leq l\left|u_{1}-u_{2}\right| \quad \text { for } \quad \text { any } \quad u_{1,2} \in \mathbb{R}, \quad x \in \Omega
$$

with a constant $l>0$.

Obviously, the stationary solutions of (1.2), if they exist, will satisfy the nonlocal elliptic equation

$$
-\sqrt{-\Delta} u+\int_{\Omega} G(x-y) F(u(y), y) d y+a u=0, a \geq 0 .
$$

We introduce the auxiliary problem

$$
\sqrt{-\Delta} u-a u=\int_{\Omega} G(x-y) F(v(y), y) d y .
$$

Let us denote $\left(f_{1}(x), f_{2}(x)\right)_{L^{2}(\Omega)}:=\int_{\Omega} f_{1}(x) \bar{f}_{2}(x) d x$, with a slight abuse of notations when these functions are not square integrable, like for example those used in the one dimensional Lemma A1 of the Appendix. The first part of the article is devoted to the studies of the case of $\Omega=\mathbb{R}^{d}$, such that the appropriate Sobolev space is equipped with the norm

$$
\|u\|_{H^{2}\left(\mathbb{R}^{d}\right)}^{2}:=\|u\|_{L^{2}\left(\mathbb{R}^{d}\right)}^{2}+\|\Delta u\|_{L^{2}\left(\mathbb{R}^{d}\right)}^{2} .
$$

The main issue for the equation above is that the operator $\sqrt{-\Delta}-a: H^{2}\left(\mathbb{R}^{d}\right) \rightarrow L^{2}\left(\mathbb{R}^{d}\right), a \geq$ 0 fails to satisfy the Fredholm property, which is the obstacle when solving problem (2.3). The similar situations but in linear problems, both self-adjoint and non self-adjoint involving non-Fredholm second or fourth order differential operators or even systems of equations with non-Fredholm operators have been studied extensively in recent years (see [19][24]). Nevertheless, we are able to prove that problem (2.3) in this case defines a map $T_{a}: H^{2}\left(\mathbb{R}^{d}\right) \rightarrow H^{2}\left(\mathbb{R}^{d}\right), a \geq 0$, which is a strict contraction under the stated technical conditions.

Theorem 1. Let $\Omega=\mathbb{R}^{d}, G(x): \mathbb{R}^{d} \rightarrow \mathbb{R}, G(x) \in W^{1,1}\left(\mathbb{R}^{d}\right)$ and Assumption 1 holds.

I) If $a>0$, then we assume that $x G(x) \in L^{1}\left(\mathbb{R}^{d}\right)$, orthogonality relations (6.6) hold for $d=1$ and (6.12) for $d=2,3$. Moreover, $\sqrt{2}(2 \pi)^{\frac{d}{2}} N_{a, d} l<1$. Then the map $T_{a} v=u$ on $H^{2}\left(\mathbb{R}^{d}\right)$ defined by problem (2.3) admits a unique fixed point $v_{a}$, which is the only stationary solution of equation (1.2) in $H^{2}\left(\mathbb{R}^{d}\right)$.

II) If $a=0$, then we assume that $x G(x) \in L^{1}\left(\mathbb{R}^{d}\right)$, orthogonality relation (6.13) holds for $d=1,2,3$ and $\sqrt{2}(2 \pi)^{\frac{d}{2}} N_{0, d} l<1$. Then the map $T_{0} v=u$ on $H^{2}\left(\mathbb{R}^{d}\right)$ defined by problem (2.3) has a unique fixed point $v_{0}$, which is the only stationary solution of equation (1.2) with $a=0$ in $H^{2}\left(\mathbb{R}^{d}\right)$.

In both cases I) and II) the fixed point $v_{a}, a \geq 0$ is nontrivial if the intersection supp $\widehat{F(0, x)} \cap \operatorname{supp} \widehat{G}$ of the supports of the Fourier images of the functions $F(0, x)$ and $G$ is a set of nonzero Lebesgue measure in $\mathbb{R}^{d}$. 
The second part of the article is devoted to the studies of the analogous equation on the finite interval with periodic boundary conditions, i.e. $\Omega=I:=[0,2 \pi]$. The appropriate functional space is as follows:

$$
H^{2}(I)=\left\{u(x): I \rightarrow \mathbb{R} \mid u(x), u^{\prime \prime}(x) \in L^{2}(I), \quad u(0)=u(2 \pi), \quad u^{\prime}(0)=u^{\prime}(2 \pi)\right\} .
$$

We define the following auxiliary constrained subspaces

$$
H_{0}^{2}(I):=\left\{u \in H^{2}(I) \mid\left(u(x), \frac{e^{ \pm i n_{0} x}}{\sqrt{2 \pi}}\right)_{L^{2}(I)}=0\right\}, n_{0} \in \mathbb{N}
$$

and

$$
H_{0,0}^{2}(I)=\left\{u \in H^{2}(I) \mid(u(x), 1)_{L^{2}(I)}=0\right\},
$$

which are Hilbert spaces as well (see e.g. Chapter 2.1 of [9]). Let us prove that problem (2.3) in this case defines a map $\tau_{a}, a \geq 0$ on the above mentioned spaces which will be a strict contraction under the given assumptions.

Theorem 2. Let $\Omega=I, G(x): I \rightarrow \mathbb{R}, G(x) \in W^{1,1}(I), G(0)=G(2 \pi), F(u, 0)=$ $F(u, 2 \pi)$ for $u \in \mathbb{R}$ and Assumption 1 holds.

I) If $a>0$ and $a \neq n, n \in \mathbb{N}$, then we assume that

$$
2 \sqrt{\pi} \mathcal{N}_{a} l<1
$$

Then the map $\tau_{a} v=u$ on $H^{2}(I)$ defined by problem (2.3) possesses a unique fixed point $v_{a}$, the only stationary solution of problem (1.2) in $H^{2}(I)$.

II) If $a=n_{0}, n_{0} \in \mathbb{N}$, assume that orthogonality conditions (6.20) hold and

$$
2 \sqrt{\pi} \mathcal{N}_{n_{0}} l<1
$$

Then the map $\tau_{n_{0}} v=u$ on $H_{0}^{2}(I)$ defined by problem (2.3) admits a unique fixed point $v_{n_{0}}$, the only stationary solution of equation (1.2) in $H_{0}^{2}(I)$.

III) If $a=0$, assume that orthogonality condition (6.21) is valid and

$$
2 \sqrt{\pi} \mathcal{N}_{0} l<1
$$

Then the map $\tau_{0} v=u$ on $H_{0,0}^{2}(I)$ defined by problem (2.3) has a unique fixed point $v_{0}$, the only stationary solution of equation (1.2) in $H_{0,0}^{2}(I)$.

In all cases I), II) and III) the fixed point $v_{a}, a \geq 0$ is nontrivial provided the Fourier coefficients $G_{n} F(0, x)_{n} \neq 0$ for a certain $n \in \mathbb{Z}$.

Remark. We use the constrained subspaces $H_{0}^{2}(I)$ and $H_{0,0}^{2}(I)$ in cases II) and III) respectively, such that the Fredholm operators

$$
\sqrt{-\frac{d^{2}}{d x^{2}}}-n_{0}: H_{0}^{2}(I) \rightarrow L^{2}(I)
$$


and

$$
\sqrt{-\frac{d^{2}}{d x^{2}}}: H_{0,0}^{2}(I) \rightarrow L^{2}(I)
$$

possess empty kernels.

Let us conclude the article with the studies of our equation on the product of spaces, where one is the finite interval with periodic boundary conditions as before and another is the whole space of dimension not exceeding two. Hence, in our notations $\Omega=I \times \mathbb{R}^{d}=$ $[0,2 \pi] \times \mathbb{R}^{d}, d=1,2$ and $x=\left(x_{1}, x_{\perp}\right)$ with $x_{1} \in I$ and $x_{\perp} \in \mathbb{R}^{d}$. The corresponding Sobolev space for the equation is $H^{2}(\Omega)$ defined as

$$
\left\{u(x): \Omega \rightarrow \mathbb{R} \mid u(x), \Delta u(x) \in L^{2}(\Omega), u\left(0, x_{\perp}\right)=u\left(2 \pi, x_{\perp}\right), u_{x_{1}}\left(0, x_{\perp}\right)=u_{x_{1}}\left(2 \pi, x_{\perp}\right)\right\},
$$

with $x_{\perp} \in \mathbb{R}^{d}$ a.e. Here $u_{x_{1}}$ denotes the derivative of $u(x)$ with respect to the first variable $x_{1}$. Analogously to the whole space case treated in Theorem 1 , the operator $\sqrt{-\Delta}-a$ : $H^{2}(\Omega) \rightarrow L^{2}(\Omega), \quad a \geq 0$ does not have the Fredholm property. We prove that equation (2.3) in such context defines a map $t_{a}: H^{2}(\Omega) \rightarrow H^{2}(\Omega), a \geq 0$, which is a strict contraction under the given technical conditions.

Theorem 3. Let $\Omega=I \times \mathbb{R}^{d}, d=1,2, \quad G(x): \Omega \rightarrow \mathbb{R}, \quad G(x) \in W^{1,1}(\Omega), \quad G\left(0, x_{\perp}\right)=$ $G\left(2 \pi, x_{\perp}\right), \quad F\left(u, 0, x_{\perp}\right)=F\left(u, 2 \pi, x_{\perp}\right)$ for $x_{\perp} \in \mathbb{R}^{d}$ a.e. and $u \in \mathbb{R}$ and Assumption 1 holds.

I) If $n_{0}<a<n_{0}+1, \quad n_{0} \in \mathbb{Z}^{+}=\mathbb{N} \cup\{0\}$ let $x_{\perp} G(x) \in L^{1}(\Omega)$, then assume that condition (6.36) holds if dimension $d=1$ and (6.37) if $d=2$ and $\sqrt{2}(2 \pi)^{\frac{d+1}{2}} M_{a} l<1$. Then the map $t_{a} v=u$ on $H^{2}(\Omega)$ defined by problem (2.3) possesses a unique fixed point $v_{a}$, the only stationary solution of equation (1.2) in $H^{2}(\Omega)$.

II) If $a=n_{0}, n_{0} \in \mathbb{N}$ let $x_{\perp}^{2} G(x) \in L^{1}(\Omega)$, conditions (6.30), (6.32) hold when dimension $d=1$ and conditions (6.31), (6.32) hold when $d=2$ and $\sqrt{2}(2 \pi)^{\frac{d+1}{2}} M_{n_{0}} l<1$. Then the map $t_{n_{0}} v=u$ on $H^{2}(\Omega)$ defined by problem (2.3) admits a unique fixed point $v_{n_{0}}$, the only stationary solution of equation (1.2) in $H^{2}(\Omega)$.

III) If $a=0$, then we assume that $x_{\perp} G(x) \in L^{1}(\Omega)$ and condition (6.29) holds. Moreover $\sqrt{2}(2 \pi)^{\frac{d+1}{2}} M_{0} l<1$. Then the map $t_{0} v=u$ on $H^{2}(\Omega)$ defined by problem (2.3) has a unique fixed point $v_{0}$, the only stationary solution of equation (1.2) in $H^{2}(\Omega)$.

In all cases I), II) and III) the fixed point $v_{a}, a \geq 0$ is nontrivial provided that for a certain $n \in \mathbb{Z}$ the intersection of supports of the Fourier images of functions supp $\widehat{F(0, x)} \cap \operatorname{supp} \widehat{G}_{n}$ is a set of nonzero Lebesgue measure in $\mathbb{R}^{d}$.

Remark. Note that the maps discussed above act on real valued functions by virtue of the assumptions on $F(u, x)$ and $G(x)$ involved in the nonlocal term of (2.3).

\section{The Whole Space Case}

Proof of Theorem 1. The argument below covers both cases I) and II) of the theorem. First we suppose that in the case of $\Omega=\mathbb{R}^{d}$ for some $v(x) \in H^{2}\left(\mathbb{R}^{d}\right)$ there exist two solutions 
$u_{1,2}(x) \in H^{2}\left(\mathbb{R}^{d}\right)$ of equation (2.3). Then their difference $w:=u_{1}-u_{2} \in H^{2}\left(\mathbb{R}^{d}\right)$ will satisfy the homogeneous equation $\sqrt{-\Delta} w=a w$. Since the $\sqrt{-\Delta}$ operator acting in the whole space does not possess any nontrivial square integrable eigenfunctions, then $w(x)=0$ a.e. in $\mathbb{R}^{d}$.

We choose arbitrarily $v(x) \in H^{2}\left(\mathbb{R}^{d}\right)$. Let us apply the standard Fourier transform (6.1) to both sides of (2.3) and obtain

$$
\widehat{u}(p)=(2 \pi)^{\frac{d}{2}} \frac{\widehat{G}(p) \widehat{f}(p)}{|p|-a},
$$

where $\widehat{f}(p)$ denotes the Fourier image of $F(v(x), x)$. Obviously, the upper bounds

$$
|\widehat{u}(p)| \leq(2 \pi)^{\frac{d}{2}} N_{a, d}|\widehat{f}(p)| \quad \text { and } \quad\left|p^{2} \widehat{u}(p)\right| \leq(2 \pi)^{\frac{d}{2}} N_{a, d}|\widehat{f}(p)|
$$

hold with $N_{a, d}<\infty$ by means of Lemma A1 of the Appendix in one dimension and via Lemma A2 for $d=2,3$ under orthogonality relations (6.6), (6.7) and (6.12), (6.13) respectively. This enables us to estimate the norm

$$
\|u\|_{H^{2}\left(\mathbb{R}^{d}\right)}^{2}=\|\widehat{u}(p)\|_{L^{2}\left(\mathbb{R}^{d}\right)}^{2}+\left\|p^{2} \widehat{u}(p)\right\|_{L^{2}\left(\mathbb{R}^{d}\right)}^{2} \leq 2(2 \pi)^{d} N_{a, d}^{2}\|F(v(x), x)\|_{L^{2}\left(\mathbb{R}^{d}\right)}^{2},
$$

which is finite by virtue of (2.1) of Assumption 1. Thus, for any $v(x) \in H^{2}\left(\mathbb{R}^{d}\right)$ there is a unique solution $u(x) \in H^{2}\left(\mathbb{R}^{d}\right)$ of equation (2.3) with its Fourier image given by (3.1) and the map $T_{a}: H^{2}\left(\mathbb{R}^{d}\right) \rightarrow H^{2}\left(\mathbb{R}^{d}\right)$ is well defined. This enables us to choose arbitrarily $v_{1,2}(x) \in H^{2}\left(\mathbb{R}^{d}\right)$ such that their images $u_{1,2}=T_{a} v_{1,2} \in H^{2}\left(\mathbb{R}^{d}\right)$ and estimate

$\left|\widehat{u}_{1}(p)-\widehat{u}_{2}(p)\right| \leq(2 \pi)^{\frac{d}{2}} N_{a, d}\left|\widehat{f}_{1}(p)-\widehat{f}_{2}(p)\right|, \quad\left|p^{2} \widehat{u}_{1}(p)-p^{2} \widehat{u}_{2}(p)\right| \leq(2 \pi)^{\frac{d}{2}} N_{a, d}\left|\widehat{f}_{1}(p)-\widehat{f}_{2}(p)\right|$,

where $\widehat{f}_{1,2}(p)$ denote the Fourier images of $F\left(v_{1,2}(x), x\right)$. For the appropriate norms of functions this yields

$$
\left\|u_{1}-u_{2}\right\|_{H^{2}\left(\mathbb{R}^{d}\right)}^{2} \leq 2(2 \pi)^{d} N_{a, d}^{2}\left\|F\left(v_{1}(x), x\right)-F\left(v_{2}(x), x\right)\right\|_{L^{2}\left(\mathbb{R}^{d}\right)}^{2} .
$$

Note that $v_{1,2}(x) \in H^{2}\left(\mathbb{R}^{d}\right) \subset L^{\infty}\left(\mathbb{R}^{d}\right), d \leq 3$ by virtue of the Sobolev embedding. By means of condition (2.2) we obtain

$$
\left\|T_{a} v_{1}-T_{a} v_{2}\right\|_{H^{2}\left(\mathbb{R}^{d}\right)} \leq \sqrt{2}(2 \pi)^{\frac{d}{2}} N_{a, d} l\left\|v_{1}-v_{2}\right\|_{H^{2}\left(\mathbb{R}^{d}\right)}
$$

with the constant in the right side of this inequality less than one due to the assumption of the theorem. Therefore, by virtue of the Fixed Point Theorem, there exists a unique function $v_{a} \in H^{2}\left(\mathbb{R}^{d}\right)$ with the property $T_{a} v_{a}=v_{a}$, which is the only stationary solution of problem (1.2) in $H^{2}\left(\mathbb{R}^{d}\right)$. Suppose $v_{a}(x)=0$ a.e. in $\mathbb{R}^{d}$. This will contradict to the assumption that the Fourier images of $G(x)$ and $F(0, x)$ do not vanish on a set of nonzero Lebesgue measure in $\mathbb{R}^{d}$. 


\section{The Problem on the Finite Interval}

Proof of Theorem 2. We will present the proof for $a>0, a \neq n, n \in \mathbb{N}$ and cases II) and III) can be treated similarly using the constrained subspaces discussed above. First let us suppose that for some $v(x) \in H^{2}(I)$ there are two solutions $u_{1,2}(x) \in H^{2}(I)$ of equation (2.3) with $\Omega=I$. Hence the function $w:=u_{1}-u_{2} \in H^{2}(I)$ will be a solution to the equation

$$
\sqrt{-\frac{d^{2}}{d x^{2}}} w=a w
$$

But when $a>0, a \neq n, n \in \mathbb{N}$, it cannot be an eigenvalue of the operator $\sqrt{-\frac{d^{2}}{d x^{2}}}$ on $H^{2}(I)$ with periodic boundary conditions. Therefore, $w(x)=0$ a.e. in $I$.

Let us choose arbitrarily $v(x) \in H^{2}(I)$. We apply the Fourier transform (6.16) to equation (2.3) considered on the interval $I$ which gives us

$$
u_{n}=\sqrt{2 \pi} \frac{G_{n} f_{n}}{|n|-a}, \quad n \in \mathbb{Z}
$$

with $f_{n}:=F(v(x), x)_{n}$. Obviously, for the transform of the second derivative we obtain

$$
\left(-u^{\prime \prime}\right)_{n}=\sqrt{2 \pi} \frac{n^{2} G_{n} f_{n}}{|n|-a}, \quad n \in \mathbb{Z},
$$

such that

$$
\left|u_{n}\right| \leq \sqrt{2 \pi} \mathcal{N}_{a}\left|f_{n}\right|, \quad\left|\left(-u^{\prime \prime}\right)_{n}\right| \leq \sqrt{2 \pi} \mathcal{N}_{a}\left|f_{n}\right|
$$

Thus, we arrive at

$$
\|u\|_{H^{2}(I)}^{2}=\sum_{n=-\infty}^{\infty}\left|u_{n}\right|^{2}+\sum_{n=-\infty}^{\infty}\left|n^{2} u_{n}\right|^{2} \leq 4 \pi \mathcal{N}_{a}^{2}\|F(v(x), x)\|_{L^{2}(I)}^{2}<\infty
$$

by means of (2.1) of Assumption 1 and Lemma A3 of the Appendix. Therefore, for an arbitrary $v(x) \in H^{2}(I)$ there is a unique $u(x) \in H^{2}(I)$ solving problem (2.3) with its Fourier image given by (4.1) and the map $\tau_{a}: H^{2}(I) \rightarrow H^{2}(I)$ is well defined. We consider arbitrary $v_{1,2}(x) \in H^{2}(I)$ with their images under the map discussed above $u_{1,2}:=\tau_{a} v_{1,2} \in H^{2}(I)$ and arrive easily at the estimate from above

$$
\begin{gathered}
\left\|u_{1}-u_{2}\right\|_{H^{2}(I)}^{2}=\sum_{n=-\infty}^{\infty}\left|u_{1 n}-u_{2 n}\right|^{2}+\sum_{n=-\infty}^{\infty}\left|n^{2}\left(u_{1 n}-u_{2 n}\right)\right|^{2} \leq \\
\leq 4 \pi \mathcal{N}_{a}^{2}\left\|F\left(v_{1}(x), x\right)-F\left(v_{2}(x), x\right)\right\|_{L^{2}(I)}^{2} .
\end{gathered}
$$

Evidently, $v_{1,2}(x) \in H^{2}(I) \subset L^{\infty}(I)$ by virtue of the Sobolev embedding. Inequality (2.2) gives us

$$
\left\|\tau_{a} v_{1}-\tau_{a} v_{2}\right\|_{H^{2}(I)} \leq 2 \sqrt{\pi} \mathcal{N}_{a} l\left\|v_{1}-v_{2}\right\|_{H^{2}(I)}
$$


The constant in the right side of this inequality is less than one due to one of our assumptions. Therefore, the Fixed Point Theorem yields the existence and uniqueness of a function $v_{a} \in$ $H^{2}(I)$ satisfying $\tau_{a} v_{a}=v_{a}$, which is the only stationary solution of problem $(1.2)$ in $H^{2}(I)$. Suppose $v_{a}(x)=0$ a.e. in $I$. Then we obtain the contradiction to our assumption that $G_{n} F(0, x)_{n} \neq 0$ for a certain $n \in \mathbb{Z}$. Note that when $a>0, a \neq n, n \in \mathbb{N}$ the argument does not rely on any orthogonality relations.

\section{The Problem on the Product of Spaces}

Proof of Theorem 3. The argument below is general for all cases I), II) and III) of the theorem. Let us suppose that there exists $v(x) \in H^{2}(\Omega)$ generating $u_{1,2}(x) \in H^{2}(\Omega)$ which solve problem (2.3). Then the difference $w(x):=u_{1}(x)-u_{2}(x) \in H^{2}(\Omega)$ will satisfy the equation

$$
\sqrt{-\Delta} w=a w
$$

in our domain $\Omega$. The partial Fourier transform applied to this problem yields

$$
\sqrt{-\Delta_{\perp}+n^{2}} w_{n}\left(x_{\perp}\right)=a w_{n}\left(x_{\perp}\right), \quad n \in \mathbb{Z},
$$

where $\Delta_{\perp}$ is the transversal Laplace operator and

$$
w(x)=\sum_{n=-\infty}^{\infty} w_{n}\left(x_{\perp}\right) \frac{e^{i n x_{1}}}{\sqrt{2 \pi}} .
$$

Obviously,

$$
\|w\|_{L^{2}(\Omega)}^{2}=\sum_{n=-\infty}^{\infty}\left\|w_{n}\right\|_{L^{2}\left(\mathbb{R}^{d}\right)}^{2} .
$$

Hence, $w_{n}\left(x_{\perp}\right) \in L^{2}\left(\mathbb{R}^{d}\right), n \in \mathbb{Z}$. The operator $\sqrt{-\Delta_{\perp}+n^{2}}$ on $L^{2}\left(\mathbb{R}^{d}\right)$ does not possess any nontrivial eigenfunctions. Therefore, $w(x)=0$ is a.e. in $\Omega$. We choose arbitrarily $v(x) \in H^{2}(\Omega)$. Let us apply the Fourier transform (6.24) to both sides of equation (2.3). This yields

$$
\widehat{u}_{n}(p)=(2 \pi)^{\frac{d+1}{2}} \frac{\widehat{G}_{n}(p) \widehat{f}_{n}(p)}{\sqrt{p^{2}+n^{2}}-a}, \quad n \in \mathbb{Z}, \quad p \in \mathbb{R}^{d}, \quad d=1,2,
$$

where $\widehat{f}_{n}(p)$ denotes the Fourier image of $F(v(x), x)$. Obviously,

$$
\left|\widehat{u}_{n}(p)\right| \leq(2 \pi)^{\frac{d+1}{2}} M_{a}\left|\widehat{f}_{n}(p)\right| \quad \text { and } \quad\left|\left(p^{2}+n^{2}\right) \widehat{u}_{n}(p)\right| \leq(2 \pi)^{\frac{d+1}{2}} M_{a}\left|\widehat{f}_{n}(p)\right|
$$

where $M_{a}<\infty$ by virtue of Lemmas A4-A6 of the Appendix under the corresponding orthogonality relations stated there. Hence

$$
\|u\|_{H^{2}(\Omega)}^{2}=\sum_{n=-\infty}^{\infty} \int_{\mathbb{R}^{d}}\left|\widehat{u}_{n}(p)\right|^{2} d p+\sum_{n=-\infty}^{\infty} \int_{\mathbb{R}^{d}}\left|\left(p^{2}+n^{2}\right) \widehat{u}_{n}(p)\right|^{2} d p \leq
$$




$$
\leq 2(2 \pi)^{d+1} M_{a}^{2}\|F(v(x), x)\|_{L^{2}(\Omega)}^{2}<\infty
$$

by virtue of (2.1) of Assumption 1. Therefore, for any $v(x) \in H^{2}(\Omega)$ there exists a unique $u(x) \in H^{2}(\Omega)$ solving problem (2.3) with its Fourier image given by (5.1) and the map $t_{a}: H^{2}(\Omega) \rightarrow H^{2}(\Omega)$ is well defined. Let us choose arbitrarily $v_{1,2} \in H^{2}(\Omega)$ such that their images under the map are $u_{1,2}:=t_{a} v_{1,2} \in H^{2}(\Omega)$ and arrive at

$$
\begin{gathered}
\left\|u_{1}-u_{2}\right\|_{H^{2}(\Omega)}^{2}=\sum_{n=-\infty}^{\infty} \int_{\mathbb{R}^{d}}\left|\widehat{u}_{1 n}(p)-\widehat{u_{2 n}}(p)\right|^{2} d p+\sum_{n=-\infty}^{\infty} \int_{\mathbb{R}^{d}}\left|\left(p^{2}+n^{2}\right)\left(\widehat{u_{1 n}}(p)-\widehat{u_{2 n}}(p)\right)\right|^{2} d p \leq \\
\leq 2(2 \pi)^{d+1} M_{a}^{2}\left\|F\left(v_{1}(x), x\right)-F\left(v_{2}(x), x\right)\right\|_{L^{2}(\Omega)}^{2} .
\end{gathered}
$$

Evidently, $v_{1,2} \in H^{2}(\Omega) \subset L^{\infty}(\Omega)$ due to the Sobolev embedding theorem. By virtue of (2.2) we easily obtain the inequality

$$
\left\|t_{a} v_{1}-t_{a} v_{2}\right\|_{H^{2}(\Omega)} \leq \sqrt{2}(2 \pi)^{\frac{d+1}{2}} M_{a} l\left\|v_{1}-v_{2}\right\|_{H^{2}(\Omega)},
$$

where the constant in the right side of it is less than one as assumed. Hence, the Fixed Point Theorem gives us the existence and uniqueness of a function $v_{a} \in H^{2}(\Omega)$ satisfying $t_{a} v_{a}=v_{a}$, which is the only stationary solution of equation $(1.2)$ in $H^{2}(\Omega)$. Suppose $v_{a}(x)=0$ a.e. in $\Omega$. This gives us the contradiction to the assumption that there exists $n \in \mathbb{Z}$ for which $\operatorname{supp} \widehat{G}_{n} \cap \operatorname{supp} \widehat{F(0, x)}$ is a set of nonzero Lebesgue measure in $\mathbb{R}^{d}$.

\section{Appendix}

Let $G(x)$ be a function, $G(x): \mathbb{R}^{d} \rightarrow \mathbb{R}, \quad d \leq 3$. We designate its standard Fourier transform via the hat symbol as

$$
\widehat{G}(p):=\frac{1}{(2 \pi)^{\frac{d}{2}}} \int_{\mathbb{R}^{d}} G(x) e^{-i p x} d x, p \in \mathbb{R}^{d} .
$$

Evidently

$$
\|\widehat{G}(p)\|_{L^{\infty}\left(\mathbb{R}^{d}\right)} \leq \frac{1}{(2 \pi)^{\frac{d}{2}}}\|G(x)\|_{L^{1}\left(\mathbb{R}^{d}\right)}
$$

and

$$
\|p \widehat{G}(p)\|_{L^{\infty}\left(\mathbb{R}^{d}\right)} \leq \frac{1}{(2 \pi)^{\frac{d}{2}}}\|\nabla G\|_{L^{1}\left(\mathbb{R}^{d}\right)} .
$$

We have $G(x)=\frac{1}{(2 \pi)^{\frac{d}{2}}} \int_{\mathbb{R}^{d}} \widehat{G}(q) e^{i q x} d q, x \in \mathbb{R}^{d}$ and define the following quantities for the technical purposes

$$
N_{a, d}:=\max \left\{\left\|\frac{\widehat{G}(p)}{|p|-a}\right\|_{L^{\infty}\left(\mathbb{R}^{d}\right)}, \quad\left\|\frac{p^{2} \widehat{G}(p)}{|p|-a}\right\|_{L^{\infty}\left(\mathbb{R}^{d}\right)}\right\}
$$


when $a>0$ and

$$
N_{0, d}:=\max \left\{\left\|\frac{\widehat{G}(p)}{|p|}\right\|_{L^{\infty}\left(\mathbb{R}^{d}\right)}, \quad\|p \widehat{G}(p)\|_{L^{\infty}\left(\mathbb{R}^{d}\right)}\right\}
$$

for $a=0$. Note that it would be sufficient to establish the boundedness of $\frac{\widehat{G}(p)}{|p|-a}$. Indeed,

$$
\frac{p^{2} \widehat{G}(p)}{|p|-a}=|p| \widehat{G}(p)+a \widehat{G}(p)+a^{2} \frac{\widehat{G}(p)}{|p|-a} .
$$

The first and the second terms in the right side of this identity will be bounded by means of inequalities (6.2) and (6.3) under the assumptions of Lemmas A1 and A2 below.

Lemma A1. Let $G(x): \mathbb{R} \rightarrow \mathbb{R}$ be such that $G(x) \in W^{1,1}(\mathbb{R})$ and $x G(x) \in L^{1}(\mathbb{R})$.

a) If $a>0$ then $N_{a, 1}<\infty$ if and only if

$$
\left(G(x), \frac{e^{ \pm i a x}}{\sqrt{2 \pi}}\right)_{L^{2}(\mathbb{R})}=0 .
$$

b) If $a=0$ then $N_{0,1}<\infty$ if and only if

$$
(G(x), 1)_{L^{2}(\mathbb{R})}=0 .
$$

Proof. In order to prove part a) of the lemma we express the function

$$
\frac{\widehat{G}(p)}{|p|-a}=\frac{\widehat{G}(p)}{p-a} \chi_{\mathbb{R}^{+}}+\frac{\widehat{G}(p)}{-p-a} \chi_{\mathbb{R}^{-}},
$$

where $\chi_{A}$ here and further down denotes the characteristic function of a set $A$. Let us use the representation formulas

$$
\widehat{G}(p)=\widehat{G}(a)+\int_{a}^{p} \frac{d \widehat{G}(q)}{d q} d q
$$

and

$$
\widehat{G}(p)=\widehat{G}(-a)+\int_{-a}^{p} \frac{d \widehat{G}(q)}{d q} d q
$$

This yields

$$
\frac{\widehat{G}(p)}{|p|-a}=\frac{\widehat{G}(a)}{p-a} \chi_{\mathbb{R}^{+}}+\frac{\int_{a}^{p} \frac{d \widehat{G}(q)}{d q} d q}{p-a} \chi_{\mathbb{R}^{+}}-\frac{\widehat{G}(-a)}{p+a} \chi_{\mathbb{R}^{-}}-\frac{\int_{-a}^{p} \frac{d \widehat{G}(q)}{d q} d q}{p+a} \chi_{\mathbb{R}^{-}} .
$$

Clearly, the definition of the Fourier transform (6.1) implies

$$
\left|\frac{d \widehat{G}(p)}{d p}\right| \leq \frac{1}{\sqrt{2 \pi}}\|x G(x)\|_{L^{1}(\mathbb{R})}<\infty
$$


as assumed. Hence, the second and the fourth terms in the right side of identity (6.9) can be estimated from above in the absolute value by $\frac{1}{\sqrt{2 \pi}}\|x G(x)\|_{L^{1}(\mathbb{R})}<\infty$. Equalities $\widehat{G}( \pm a)=0$ are equivalent to orthogonality conditions (6.6). When $a=0$, we express

$$
\widehat{G}(p)=\widehat{G}(0)+\int_{0}^{p} \frac{d \widehat{G}(q)}{d q} d q .
$$

This yields

$$
\frac{\widehat{G}(p)}{|p|}=\frac{\widehat{G}(0)}{|p|}+\frac{\int_{0}^{p} \frac{d \widehat{G}(q)}{d q} d q}{|p|} .
$$

The second term in the right side of (6.11) can be easily bounded above in the absolute value by $\frac{1}{\sqrt{2 \pi}}\|x G(x)\|_{L^{1}(\mathbb{R})}<\infty$. The equality $\widehat{G}(0)=0$ is equivalent to orthogonality relation $(6.7)$.

The statement above can be generalized to higher dimensions in the following proposition.

Lemma A2. Let $G(x): \mathbb{R}^{d} \rightarrow \mathbb{R}$ be such that $G(x) \in W^{1,1}\left(\mathbb{R}^{d}\right), x G(x) \in L^{1}\left(\mathbb{R}^{d}\right), d=$ 2,3 .

a) If $a>0$, then $N_{a, d}<\infty$ if and only if

$$
\left(G(x), \frac{e^{i p x}}{(2 \pi)^{\frac{d}{2}}}\right)_{L^{2}\left(\mathbb{R}^{d}\right)}=0 \quad \text { for } \quad p \in S_{a}^{d} \quad \text { a.e. }
$$

b) If $a=0$, then $N_{0, d}<\infty$ if and only if

$$
(G(x), 1)_{L^{2}\left(\mathbb{R}^{d}\right)}=0
$$

Proof. To prove part a) of the lemma, we use the representation formula

$$
\widehat{G}(p)=\widehat{G}(a, \omega)+\int_{a}^{|p|} \frac{\partial \widehat{G}}{\partial s}(s, \omega) d s .
$$

Here and below $\omega$ denotes the angle variables on the sphere. This yields

$$
\frac{\widehat{G}(p)}{|p|-a}=\frac{\widehat{G}(a, \omega)}{|p|-a}+\frac{\int_{a}^{|p|} \frac{\partial \widehat{G}}{\partial s}(s, \omega) d s}{|p|-a} .
$$

Clearly, the definition of the Fourier transform (6.1) gives us

$$
\left|\frac{\partial \widehat{G}(|p|, \omega)}{\partial|p|}\right| \leq \frac{1}{(2 \pi)^{\frac{d}{2}}}\|x G(x)\|_{L^{1}\left(\mathbb{R}^{d}\right)}<\infty
$$


due to one of our assumptions. This enables us to estimate from above the second term in the right side of (6.14) in the absolute value by $\frac{1}{(2 \pi)^{\frac{d}{2}}}\|x G(x)\|_{L^{1}\left(\mathbb{R}^{d}\right)}<\infty$. The equality $\widehat{G}(a, \omega)=0$ a.e. is equivalent to orthogonality condition (6.12).

When $a=0$, we use the identity

$$
\widehat{G}(p)=\widehat{G}(0)+\int_{0}^{|p|} \frac{\partial \widehat{G}}{\partial s}(s, \omega) d s .
$$

This yields

$$
\frac{\widehat{G}(p)}{|p|}=\frac{\widehat{G}(0)}{|p|}+\frac{\int_{0}^{|p|} \frac{\partial \widehat{G}}{\partial s}(s, \omega) d s}{|p|} .
$$

Evidently, the second term in the right side of (6.15) can be bounded from above in the absolute value by $\frac{1}{(2 \pi)^{\frac{d}{2}}}\|x G(x)\|_{L^{1}\left(\mathbb{R}^{d}\right)}$. Equality $\widehat{G}(0)=0$ is equivalent to orthogonality relation $(6.13)$.

Let the function $G(x): I \rightarrow \mathbb{R}, \quad G(0)=G(2 \pi)$ and its Fourier image on the finite interval be given by

$$
G_{n}:=\int_{0}^{2 \pi} G(x) \frac{e^{-i n x}}{\sqrt{2 \pi}} d x, \quad n \in \mathbb{Z}
$$

and $G(x)=\sum_{n=-\infty}^{\infty} G_{n} \frac{e^{i n x}}{\sqrt{2 \pi}}$. Then we have the bounds

$$
\left\|G_{n}\right\|_{l^{\infty}} \leq \frac{1}{\sqrt{2 \pi}}\|G(x)\|_{L^{1}(I)}, \quad\left\|n G_{n}\right\|_{l^{\infty}} \leq \frac{1}{\sqrt{2 \pi}}\left\|G^{\prime}(x)\right\|_{L^{1}(I)}
$$

Analogously to the whole space case, let us define

$$
\mathcal{N}_{a}:=\max \left\{\left\|\frac{G_{n}}{|n|-a}\right\|_{l^{\infty}}, \quad\left\|\frac{n^{2} G_{n}}{|n|-a}\right\|_{l^{\infty}}\right\}
$$

for $a>0$. In the case of $a=0$

$$
\mathcal{N}_{0}:=\max \left\{\left\|\frac{G_{n}}{n}\right\|_{l^{\infty}}, \quad\left\|n G_{n}\right\|_{l^{\infty}}\right\} .
$$

Note that it is sufficient to prove the boundedness of $\frac{G_{n}}{|n|-a}$. Indeed,

$$
\frac{n^{2} G_{n}}{|n|-a}=|n| G_{n}+a G_{n}+\frac{a^{2} G_{n}}{|n|-a}
$$


The first and the second terms in the right side of the equality above are bounded by means of (6.17) under the assumptions of the lemma below. We have the following trivial proposition.

Lemma A3. Let $G(x): I \rightarrow \mathbb{R}$ be such that $G(x) \in W^{1,1}(I)$ and $G(0)=G(2 \pi)$.

a) If $a>0$ and $a \neq n, n \in \mathbb{N}$, then $\mathcal{N}_{a}<\infty$.

b) If $a=n_{0}, n_{0} \in \mathbb{N}$, then $\mathcal{N}_{n_{0}}<\infty$ if and only if

$$
\left(G(x), \frac{e^{ \pm i n_{0} x}}{\sqrt{2 \pi}}\right)_{L^{2}(I)}=0 .
$$

c) If $a=0$, then $\mathcal{N}_{0}<\infty$ if and only if

$$
(G(x), 1)_{L^{2}(I)}=0 .
$$

Proof. In the case when $a>0, a \neq n, n \in \mathbb{N}$, let $\delta>0$ be the distance on the real line from $a$ to the nearest nonnegative integer. Then, by means of (6.17) we have the estimate for $n \in \mathbb{Z}$

$$
\left|\frac{G_{n}}{|n|-a}\right| \leq \frac{1}{\sqrt{2 \pi} \delta}\|G(x)\|_{L^{1}(I)}<\infty
$$

as assumed. When $a=n_{0}, n_{0} \in \mathbb{N}$, we express

$$
\frac{G_{n}}{|n|-n_{0}}=\frac{G_{n}}{|n|-n_{0}} \chi_{\left\{n \in \mathbb{Z}, n \neq \pm n_{0}\right\}}+\frac{G_{n}}{|n|-n_{0}} \chi_{\left\{n \in \mathbb{Z}, n= \pm n_{0}\right\}}
$$

Clearly, the first term in the right side of (6.22) can be bounded from above in the absolute value via (6.17) by $\frac{1}{\sqrt{2 \pi}}\|G(x)\|_{L^{1}(I)}<\infty$. Equalities $G_{ \pm n_{0}}=0$ are equivalent to orthogonality relations (6.20). Finally, when $a=0$, we have

$$
\frac{G_{n}}{n}=\frac{G_{n}}{n} \chi_{\{n \in \mathbb{Z}, n \neq 0\}}+\frac{G_{n}}{n} \chi_{\{n \in \mathbb{Z}, n=0\}} .
$$

Evidently, the first term in the right side of (6.23) can be estimated from above in the absolute value by means of (6.17) by $\frac{1}{\sqrt{2 \pi}}\|G(x)\|_{L^{1}(I)}<\infty$. Equality $G_{0}=0$ is equivalent to orthogonality condition (6.21).

Let $G(x)$ be a function on the product of spaces treated in Theorem $3, G(x): \Omega=$ $I \times \mathbb{R}^{d} \rightarrow \mathbb{R}, d=1,2, G\left(0, x_{\perp}\right)=G\left(2 \pi, x_{\perp}\right)$ for $x_{\perp} \in \mathbb{R}^{d}$ a.e. and its Fourier image on the product of spaces equals to

$$
\widehat{G}_{n}(p):=\frac{1}{(2 \pi)^{\frac{d+1}{2}}} \int_{\mathbb{R}^{d}} d x_{\perp} e^{-i p x_{\perp}} \int_{0}^{2 \pi} G\left(x_{1}, x_{\perp}\right) e^{-i n x_{1}} d x_{1}, \quad p \in \mathbb{R}^{d}, n \in \mathbb{Z} .
$$

Thus

$$
\left\|\widehat{G}_{n}(p)\right\|_{L_{n, p}^{\infty}}:=\sup _{\left\{p \in \mathbb{R}^{d}, n \in \mathbb{Z}\right\}}\left|\widehat{G}_{n}(p)\right| \leq \frac{1}{(2 \pi)^{\frac{d+1}{2}}}\|G(x)\|_{L^{1}(\Omega)}
$$


and $G(x)=\frac{1}{(2 \pi)^{\frac{d+1}{2}}} \sum_{n=-\infty}^{\infty} \int_{\mathbb{R}^{d}} \widehat{G}_{n}(p) e^{i p x_{\perp}} e^{i n x_{1}} d p$. Let us also consider the Fourier transform only in the first variable, such that

$$
G_{n}\left(x_{\perp}\right):=\int_{0}^{2 \pi} G\left(x_{1}, x_{\perp}\right) \frac{e^{-i n x_{1}}}{\sqrt{2 \pi}} d x_{1}, \quad n \in \mathbb{Z} .
$$

We define $\xi_{n}^{a}(p):=\frac{\widehat{G}_{n}(p)}{\sqrt{p^{2}+n^{2}}-a}$ and denote

$$
M_{a}:=\max \left\{\left\|\xi_{n}^{a}(p)\right\|_{L_{n, p}^{\infty}}, \quad\left\|\left(p^{2}+n^{2}\right) \xi_{n}^{a}(p)\right\|_{L_{n, p}^{\infty}}\right\}
$$

when $a>0$ and

$$
M_{0}:=\max \left\{\left\|\frac{\widehat{G}_{n}(p)}{\sqrt{p^{2}+n^{2}}}\right\|_{L_{n, p}^{\infty}}, \quad\left\|\sqrt{p^{2}+n^{2}} \widehat{G}_{n}(p)\right\|_{L_{n, p}^{\infty}}\right\}
$$

for $a=0$ with the momentum vector $p \in \mathbb{R}^{d}$. Note that it would be sufficient to prove the boundedness of the expression $\xi_{n}^{a}(p)$. Indeed,

$$
\left(p^{2}+n^{2}\right) \xi_{n}^{a}(p)=\sqrt{p^{2}+n^{2}} \widehat{G}_{n}(p)+a \widehat{G}_{n}(p)+a^{2} \xi_{n}^{a}(p) .
$$

The second term in the right side of (6.28) is bounded via (6.25) since $G(x) \in W^{1,1}(\Omega)$ as assumed in Lemmas A4-A6 below. Clearly,

$$
\left|n \widehat{G}_{n}(p)\right| \leq \frac{1}{(2 \pi)^{\frac{d+1}{2}}}\left\|\frac{\partial G}{\partial x_{1}}\right\|_{L^{1}(\Omega)}<\infty, \quad\left|p \widehat{G}_{n}(p)\right| \leq \frac{1}{(2 \pi)^{\frac{d+1}{2}}}\left\|\nabla_{x_{\perp}} G(x)\right\|_{L^{1}(\Omega)}<\infty,
$$

such that the first term in the right side of (6.28) is bounded as well. Here $\nabla_{x_{\perp}}$ is the gradient with respect to $x_{\perp}$.

Lemma A4. Let $G(x): \Omega \rightarrow \mathbb{R}$ be such that $G(x) \in W^{1,1}(\Omega), x_{\perp} G(x) \in L^{1}(\Omega)$ and $G\left(0, x_{\perp}\right)=G\left(2 \pi, x_{\perp}\right)$ for $x_{\perp} \in \mathbb{R}^{d}$ a.e., $d=1,2$. Then $M_{0}<\infty$ if and only if

$$
(G(x), 1)_{L^{2}(\Omega)}=0 .
$$

Proof. We expand

$$
\xi_{n}^{0}(p)=\xi_{n}^{0}(p) \chi_{\left\{p \in \mathbb{R}^{d}, n=0\right\}}+\xi_{n}^{0}(p) \chi_{\left\{p \in \mathbb{R}^{d}, n \in \mathbb{Z}, n \neq 0\right\}} .
$$

The second term in the right side of this identity can be bounded from above in the absolute value via (6.25) by $\frac{1}{(2 \pi)^{\frac{d+1}{2}}}\|G(x)\|_{L^{1}(\Omega)}<\infty$ as assumed. Obviously, we have the bounds on the norms

$$
\left\|G_{0}\left(x_{\perp}\right)\right\|_{L^{1}\left(\mathbb{R}^{d}\right)} \leq \frac{1}{\sqrt{2 \pi}}\|G(x)\|_{L^{1}(\Omega)}<\infty, \quad\left\|x_{\perp} G_{0}\left(x_{\perp}\right)\right\|_{L^{1}\left(\mathbb{R}^{d}\right)} \leq \frac{1}{\sqrt{2 \pi}}\left\|x_{\perp} G(x)\right\|_{L^{1}(\Omega)}<\infty
$$


due to the assumptions of the lemma. Therefore, the remaining term $\frac{\widehat{G}_{0}(p)}{|p|} \in L^{\infty}\left(\mathbb{R}^{d}\right)$ if and only if orthogonality relation (6.29) holds, which is guaranteed when $d=1$ by Lemma A1 and for dimension $d=2$ by Lemma A2.

Next we turn our attention to the cases where the parameter $a$ does not vanish.

Lemma A5. Let $G(x): \Omega \rightarrow \mathbb{R}$ be such that $G(x) \in W^{1,1}(\Omega), \quad x_{\perp}^{2} G(x) \in L^{1}(\Omega)$ and $G\left(0, x_{\perp}\right)=G\left(2 \pi, x_{\perp}\right)$ for $x_{\perp} \in \mathbb{R}^{d}$ a.e., $d=1,2$ and $a=n_{0}, n_{0} \in \mathbb{N}$. Then $M_{n_{0}}<\infty$ if and only if

$$
\begin{gathered}
\left(G\left(x_{1}, x_{\perp}\right), \frac{e^{i n x_{1}}}{\sqrt{2 \pi}} \frac{e^{ \pm i \sqrt{n_{0}^{2}-n^{2}} x_{\perp}}}{\sqrt{2 \pi}}\right)_{L^{2}(\Omega)}=0, \quad|n| \leq n_{0}-1, \quad d=1, \\
\left(G\left(x_{1}, x_{\perp}\right), \frac{e^{i n x_{1}}}{\sqrt{2 \pi}} \frac{e^{i p x_{\perp}}}{2 \pi}\right)_{L^{2}(\Omega)}=0, \quad p \in S_{\sqrt{n_{0}^{2}-n^{2}}}^{2} \quad \text { a.e., } \quad|n| \leq n_{0}-1, \quad d=2, \\
\left(G\left(x_{1}, x_{\perp}\right), \frac{e^{ \pm i n_{0} x_{1}}}{\sqrt{2 \pi}}\right)_{L^{2}(\Omega)}=0, \quad\left(G\left(x_{1}, x_{\perp}\right), \frac{e^{ \pm i n_{0} x_{1}}}{\sqrt{2 \pi}} x_{\perp}, k\right)_{L^{2}(\Omega)}=0, \quad 1 \leq k \leq d .
\end{gathered}
$$

Proof. Let us use the representation of the function $\xi_{n}^{n_{0}}(p), n \in \mathbb{Z}, p \in \mathbb{R}^{d}$ as the sum

$$
\xi_{n}^{n_{0}}(p) \chi_{\left\{p \in \mathbb{R}^{d},|n| \geq n_{0}+1\right\}}+\xi_{n}^{n_{0}}(p) \chi_{\left\{p \in \mathbb{R}^{d},|n| \leq n_{0}\right\}} .
$$

Evidently, $\left|\xi_{n}^{n_{0}}(p) \chi_{\left\{p \in \mathbb{R}^{d},|n| \geq n_{0}+1\right\}}\right| \leq\left\|\widehat{G}_{n}(p)\right\|_{L_{n, p}^{\infty},}$, which is bounded by means of (6.25). Hence, one needs to estimate

$$
\xi_{n}^{n_{0}}(p) \chi_{\left\{p \in \mathbb{R}^{d},|n|<n_{0}\right\}}+\xi_{n}^{n_{0}}(p) \chi_{\left\{p \in \mathbb{R}^{d},|n|=n_{0}\right\}} .
$$

Apparently, by virtue of $(6.25)$

$$
\left|\xi_{n}^{n_{0}}(p) \chi_{\left\{|p|>1,|n|=n_{0}\right\}}\right| \leq \frac{1}{\sqrt{1+n_{0}^{2}}-n_{0}} \frac{1}{(2 \pi)^{\frac{d+1}{2}}}\|G(x)\|_{L^{1}(\Omega)}<\infty
$$

as assumed. Let us first treat the case for the dimension $d=1$. We use the representation formula

$$
\widehat{G}_{n}(p)=\widehat{G}_{n}(0)+p \frac{d \widehat{G}_{n}}{d p}(0)+\int_{0}^{p}\left(\int_{0}^{s} \frac{d^{2} \widehat{G}_{n}(q)}{d q^{2}} d q\right) d s .
$$

This gives us the sum

$$
\left[\frac{\widehat{G}_{n}(0)}{\sqrt{p^{2}+n^{2}}-n_{0}}+\frac{p \frac{d \widehat{G}_{n}}{d p}(0)}{\sqrt{p^{2}+n^{2}}-n_{0}}+\frac{\int_{0}^{p}\left(\int_{0}^{s} \frac{d^{2} \widehat{G}_{n}(q)}{d q^{2}} d q\right) d s}{\sqrt{p^{2}+n^{2}}-n_{0}}\right] \chi_{\left\{|p| \leq 1,|n|=n_{0}\right\}} .
$$

Clearly,

$$
\left|\frac{d^{2} \widehat{G}_{n}(p)}{d p^{2}}\right| \leq \frac{1}{2 \pi}\left\|x_{\perp}^{2} G\left(x_{1}, x_{\perp}\right)\right\|_{L^{1}(\Omega)}<\infty
$$


due to one of our assumptions. Apparently, the last term in (6.33) can be estimated from above in the absolute value by

$$
\frac{1}{4 \pi}\left\|x_{\perp}^{2} G\left(x_{1}, x_{\perp}\right)\right\|_{L^{1}(\Omega)} \frac{p^{2}}{\sqrt{p^{2}+n_{0}^{2}}-n_{0}} \chi_{\left\{|p| \leq 1,|n|=n_{0}\right\}} \leq C .
$$

Here and below $C$ denotes a finite, positive constant. Equalities

$$
\widehat{G}_{n}(0)=0, \quad \frac{d \widehat{G}_{n}}{d p}(0)=0
$$

for $n= \pm n_{0}$ are equivalent to orthogonality relations (6.32) in one dimension. Hence, it remains to study the sum

$$
\xi_{n}^{n_{0}}(p) \chi_{\left\{p \in \mathbb{R}^{+},|n|<n_{0}\right\}}+\xi_{n}^{n_{0}}(p) \chi_{\left\{p \in \mathbb{R}^{-},|n|<n_{0}\right\}}
$$

Let us use the identity

$$
\widehat{G}_{n}(p)=\widehat{G}_{n}\left(\sqrt{n_{0}^{2}-n^{2}}\right)+\int_{\sqrt{n_{0}^{2}-n^{2}}}^{p} \frac{d \widehat{G}_{n}(s)}{d s} d s
$$

for $|n|<n_{0}$. This enables us to write the first term in sum (6.34) as

$$
\left[\frac{\widehat{G}_{n}\left(\sqrt{n_{0}^{2}-n^{2}}\right)}{\sqrt{p^{2}+n^{2}}-n_{0}}+\frac{\int^{p} \sqrt{n_{0}^{2}-n^{2}} \frac{d \widehat{G}_{n}(s)}{d s} d s}{\sqrt{p^{2}+n^{2}}-n_{0}}\right] \chi_{\left\{p \in \mathbb{R}^{+},|n|<n_{0}\right\}} .
$$

The definition of our mixed Fourier transform (6.24) allows us to estimate from above in the absolute value the second term in this sum by

$$
\frac{1}{2 \pi}\left\|x_{\perp} G\left(x_{1}, x_{\perp}\right)\right\|_{L^{1}(\Omega)} \frac{\left|p-\sqrt{n_{0}^{2}-n^{2}}\right|}{\left|\sqrt{p^{2}+n^{2}}-n_{0}\right|} \chi_{\left\{p \in \mathbb{R}^{+},|n|<n_{0}\right\}} \leq C .
$$

Note that $G(x) \in L^{1}(\Omega)$ and $x_{\perp}^{2} G(x) \in L^{1}(\Omega)$ as assumed in the lemma, which implies $x_{\perp} G(x) \in L^{1}(\Omega)$. We also express

$$
\widehat{G}_{n}(p)=\widehat{G}_{n}\left(-\sqrt{n_{0}^{2}-n^{2}}\right)+\int_{-\sqrt{n_{0}^{2}-n^{2}}}^{p} \frac{d \widehat{G}_{n}(s)}{d s} d s
$$

with $|n|<n_{0}$. Thus, the second term in (6.34) equals to

$$
\left[\frac{\widehat{G}_{n}\left(-\sqrt{n_{0}^{2}-n^{2}}\right)}{\sqrt{p^{2}+n^{2}}-n_{0}}+\frac{\int_{-\sqrt{n_{0}^{2}-n^{2}}}^{p} \frac{d \widehat{G}_{n}(s)}{d s} d s}{\sqrt{p^{2}+n^{2}}-n_{0}}\right] \chi_{\left\{p \in \mathbb{R}^{-},|n|<n_{0}\right\}} .
$$


Evidently, the second term in this sum can be bounded from above in the absolute value by

$$
\frac{1}{2 \pi}\left\|x_{\perp} G\left(x_{1}, x_{\perp}\right)\right\|_{L^{1}(\Omega)} \frac{\left|p+\sqrt{n_{0}^{2}-n^{2}}\right|}{\left|\sqrt{p^{2}+n^{2}}-n_{0}\right|} \chi_{\left\{p \in \mathbb{R}^{-},|n|<n_{0}\right\}} \leq C .
$$

Equalities

$$
\widehat{G}_{n}\left( \pm \sqrt{n_{0}^{2}-n^{2}}\right)=0, \quad|n|<n_{0}
$$

are equivalent to orthogonality relations (6.30). Then we turn our attention to the case of dimension $d=2$. Let us use the representation formula

$$
\widehat{G}_{n}(p)=\widehat{G}_{n}(0)+\frac{\partial \widehat{G}_{n}(0, \omega)}{\partial|p|}|p|+\int_{0}^{|p|}\left(\int_{0}^{s} \frac{\partial^{2} \widehat{G}_{n}(q, \omega)}{\partial q^{2}} d q\right) d s .
$$

Thus our goal is to study the following sum

$$
\left[\frac{\widehat{G}_{n}(0)}{\sqrt{p^{2}+n^{2}}-n_{0}}+\frac{\frac{\partial \widehat{G}_{n}(0, \omega)}{\partial|p|}|p|}{\sqrt{p^{2}+n^{2}}-n_{0}}+\frac{\int_{0}^{|p|}\left(\int_{0}^{s} \frac{\partial^{2} \widehat{G}_{n}(q, \omega)}{\partial q^{2}} d q\right) d s}{\sqrt{p^{2}+n^{2}}-n_{0}}\right] \chi_{\left\{|p| \leq 1,|n|=n_{0}\right\}} .
$$

Clearly, we have

$$
\left|\frac{\partial^{2} \widehat{G}_{n}(|p|, \omega)}{\partial|p|^{2}}\right| \leq \frac{1}{(2 \pi)^{\frac{3}{2}}}\left\|x_{\perp}^{2} G(x)\right\|_{L^{1}(\Omega)}<\infty
$$

due to one of our assumptions. Hence, the last term in (6.35) can be bounded from above in the absolute value by

$$
\frac{1}{2(2 \pi)^{\frac{3}{2}}}\left\|x_{\perp}^{2} G(x)\right\|_{L^{1}(\Omega)} \frac{|p|^{2}}{\sqrt{p^{2}+n_{0}^{2}}-n_{0}} \chi_{\left\{|p| \leq 1,|n|=n_{0}\right\}} \leq C .
$$

Equalities

$$
\widehat{G}_{n}(0)=0, \quad \frac{\partial \widehat{G}_{n}}{\partial|p|}(0, \omega)=0, \quad n= \pm n_{0}
$$

are equivalent to orthogonality conditions (6.32) when dimension $d=2$. Finally, it remains to investigate the term

$$
\xi_{n}^{n_{0}}(p) \chi_{\left\{p \in \mathbb{R}^{2},|n|<n_{0}\right\}} .
$$

Let us use the representation formula for $|n|<n_{0}$,

$$
\widehat{G}_{n}(p)=\widehat{G}_{n}\left(\sqrt{n_{0}^{2}-n^{2}}, \omega\right)+\int_{\sqrt{n_{0}^{2}-n^{2}}}^{|p|} \frac{\partial \widehat{G}_{n}}{\partial s}(s, \omega) d s,
$$

which yields

$$
\left[\frac{\widehat{G}_{n}\left(\sqrt{n_{0}^{2}-n^{2}}, \omega\right)}{\sqrt{p^{2}+n^{2}}-n_{0}}+\frac{\int_{\sqrt{n_{0}^{2}-n^{2}}}^{|p|} \frac{\partial \widehat{G}_{n}}{\partial s}(s, \omega) d s}{\sqrt{p^{2}+n^{2}}-n_{0}}\right] \chi_{\left\{p \in \mathbb{R}^{2},|n|<n_{0}\right\}} .
$$


Apparently, the second term in this sum can be estimated from above in the absolute value by

$$
\frac{1}{(2 \pi)^{\frac{3}{2}}}\left\|x_{\perp} G(x)\right\|_{L^{1}(\Omega)} \frac{|| p\left|-\sqrt{n_{0}^{2}-n^{2}}\right|}{\left|\sqrt{p^{2}+n^{2}}-n_{0}\right|} \chi_{\left\{p \in \mathbb{R}^{2},|n|<n_{0}\right\}} \leq C .
$$

Equality

$$
\widehat{G}_{n}\left(\sqrt{n_{0}^{2}-n^{2}}, \omega\right)=0, \quad|n|<n_{0}
$$

is equivalent to orthogonality relation (6.31) when dimension $d=2$.

Let us conclude the article with the studies of the case where the parameter $a$ is located on an open interval between two consecutive nonnegative integers.

Lemma A6. Let $G(x): \Omega \rightarrow \mathbb{R}$ be such that $G(x) \in W^{1,1}(\Omega), \quad x_{\perp} G(x) \in L^{1}(\Omega)$ and $G\left(0, x_{\perp}\right)=G\left(2 \pi, x_{\perp}\right)$ for $x_{\perp} \in \mathbb{R}^{d}$ a.e., $d=1,2$ and $n_{0}<a<n_{0}+1, n_{0} \in \mathbb{Z}^{+}=\mathbb{N} \cup\{0\}$. Then $M_{a}<\infty$ if and only if

$$
\begin{gathered}
\left(G\left(x_{1}, x_{\perp}\right), \frac{e^{i n x_{1}}}{\sqrt{2 \pi}} \frac{e^{ \pm i \sqrt{a^{2}-n^{2}} x_{\perp}}}{\sqrt{2 \pi}}\right)_{L^{2}(\Omega)}=0, \quad|n| \leq n_{0}, \quad d=1, \\
\left(G\left(x_{1}, x_{\perp}\right), \frac{e^{i n x_{1}}}{\sqrt{2 \pi}} \frac{e^{i p x_{\perp}}}{2 \pi}\right)_{L^{2}(\Omega)}=0, \quad p \in S_{\sqrt{a^{2}-n^{2}}}^{2} \text { a.e., } \quad|n| \leq n_{0}, \quad d=2 .
\end{gathered}
$$

Proof. We expand $\xi_{n}^{a}(p)$ as the sum of the two terms

$$
\xi_{n}^{a}(p) \chi_{\left\{p \in \mathbb{R}^{d}, n \in \mathbb{Z},|n| \geq n_{0}+1\right\}}+\xi_{n}^{a}(p) \chi_{\left\{p \in \mathbb{R}^{d}, n \in \mathbb{Z},|n| \leq n_{0}\right\}} .
$$

Clearly, via (6.25) the absolute value of the first one is bounded from above by

$$
\frac{1}{(2 \pi)^{\frac{d+1}{2}}} \frac{\|G(x)\|_{L^{1}(\Omega)}}{n_{0}+1-a}<\infty
$$

by means of our assumptions. Let us first consider the case for the dimension $d=1$. Then it remains to study

$$
\xi_{n}^{a}(p) \chi_{\left\{p \in \mathbb{R}^{+},|n| \leq n_{0}\right\}}+\xi_{n}^{a}(p) \chi_{\left\{p \in \mathbb{R}^{-},|n| \leq n_{0}\right\}} .
$$

To treat the first term in sum (6.38), we use the representation formula

$$
\widehat{G}_{n}(p)=\widehat{G}_{n}\left(\sqrt{a^{2}-n^{2}}\right)+\int_{\sqrt{a^{2}-n^{2}}}^{p} \frac{d \widehat{G}_{n}(s)}{d s} d s, \quad|n| \leq n_{0} .
$$

This yields

$$
\left[\frac{\widehat{G}_{n}\left(\sqrt{a^{2}-n^{2}}\right)}{\sqrt{p^{2}+n^{2}}-a}+\frac{\int_{\sqrt{a^{2}-n^{2}}}^{p} \frac{d \widehat{G}_{n}(s)}{d s} d s}{\sqrt{p^{2}+n^{2}}-a}\right] \chi_{\left\{p \in \mathbb{R}^{+},|n| \leq n_{0}\right\}} .
$$


Apparently, the second term in this sum can be bounded from above in the absolute value by

$$
\frac{1}{2 \pi}\left\|x_{\perp} G(x)\right\|_{L^{1}(\Omega)} \frac{\left|p-\sqrt{a^{2}-n^{2}}\right|}{\left|\sqrt{p^{2}+n^{2}}-a\right|} \chi_{\left\{p \in \mathbb{R}^{+},|n| \leq n_{0}\right\}} \leq C .
$$

Similarly, to study the second term in (6.38), we apply the formula

$$
\widehat{G}_{n}(p)=\widehat{G}_{n}\left(-\sqrt{a^{2}-n^{2}}\right)+\int_{-\sqrt{a^{2}-n^{2}}}^{p} \frac{d \widehat{G}_{n}(s)}{d s} d s, \quad|n| \leq n_{0} .
$$

This gives us

$$
\left[\frac{\widehat{G}_{n}\left(-\sqrt{a^{2}-n^{2}}\right)}{\sqrt{p^{2}+n^{2}}-a}+\frac{\int_{-\sqrt{a^{2}-n^{2}}}^{p} \frac{d \widehat{G}_{n}(s)}{d s} d s}{\sqrt{p^{2}+n^{2}}-a}\right] \chi_{\left\{p \in \mathbb{R}^{-},|n| \leq n_{0}\right\}} .
$$

Evidently, the second term in such sum can be estimated from above in the absolute value by

$$
\frac{1}{2 \pi}\left\|x_{\perp} G(x)\right\|_{L^{1}(\Omega)} \frac{\left|p+\sqrt{a^{2}-n^{2}}\right|}{\left|\sqrt{p^{2}+n^{2}}-a\right|} \chi_{\left\{p \in \mathbb{R}^{-},|n| \leq n_{0}\right\}} \leq C .
$$

Equalities

$$
\widehat{G}_{n}\left( \pm \sqrt{a^{2}-n^{2}}\right)=0, \quad|n| \leq n_{0}
$$

are equivalent to orthogonality conditions (6.36) when dimension $d=1$. Finally, let us turn our attention to the case when $d=2$. Then we express

$$
\widehat{G}_{n}(p)=\widehat{G}_{n}\left(\sqrt{a^{2}-n^{2}}, \omega\right)+\int_{\sqrt{a^{2}-n^{2}}}^{|p|} \frac{\partial \widehat{G}_{n}(s, \omega)}{\partial s} d s, \quad|n| \leq n_{0} .
$$

Thus, we arrive at

$$
\left[\frac{\widehat{G}_{n}\left(\sqrt{a^{2}-n^{2}}, \omega\right)}{\sqrt{p^{2}+n^{2}}-a}+\frac{\int_{\sqrt{a^{2}-n^{2}}}^{|p|} \frac{\partial \widehat{G}_{n}(s, \omega)}{\partial s} d s}{\sqrt{p^{2}+n^{2}}-a}\right] \chi_{\left\{p \in \mathbb{R}^{2},|n| \leq n_{0}\right\}} .
$$

The second term in such sum can be trivially bounded from above by

$$
\frac{1}{(2 \pi)^{\frac{3}{2}}}\left\|x_{\perp} G(x)\right\|_{L^{1}(\Omega)} \frac{|| p\left|-\sqrt{a^{2}-n^{2}}\right|}{\left|\sqrt{p^{2}+n^{2}}-a\right|} \chi_{\left\{p \in \mathbb{R}^{2},|n| \leq n_{0}\right\}} \leq C .
$$

Equality

$$
\widehat{G}_{n}\left(\sqrt{a^{2}-n^{2}}, \omega\right)=0, \quad|n| \leq n_{0}
$$

is equivalent to orthogonality relations (6.37) when dimension $d=2$.

\section{Acknowledgments}

The second author was supported by the grant of Russian Science Foundation, project no. 15-11-00029 and by the program PICS CNRS 6583 Matbio. 


\section{References}

[1] M.S. Agranovich. Elliptic boundary problems, Encyclopaedia Math. Sci., vol. 79, Partial Differential Equations, IX, Springer, Berlin, 1997, pp. 1-144

[2] N. Apreutesei, N. Bessonov, V. Volpert, V. Vougalter. Spatial Structures and Generalized Travelling Waves for an Integro-Differential Equation, Discrete Contin. Dyn. Syst. Ser. B, 13, 3 (2010), 537-557

[3] H. Berestycki, G. Nadin, B. Perthame, L. Ryzhik. The non-local FisherKPP equation: travelling waves and steady states, Nonlinearity, 22, 12 (2009), 2813-2844

[4] B. Carreras, V. Lynch, G. Zaslavsky. Anomalous diffusion and exit time distribution of particle tracers in plasma turbulence model, Phys. Plasmas, 8 (2001), 5096-5103

[5] A. Ducrot, M. Marion and V. Volpert. Systemes de réaction-diffusion sans propriété de Fredholm, CRAS, 340, 9 (2005), 659-664

[6] A. Ducrot, M. Marion and V. Volpert. Reaction-diffusion problems with non Fredholm operators, Adv. Differential Equations, 13, 11-12 (2008), 1151-1192

[7] A. Ducrot, M. Marion and V. Volpert. Reaction-diffusion waves (with the Lewis number different from 1). Publibook, Paris, 2009, 113 pp.

[8] S. Genieys, V. Volpert, P. Auger. Pattern and waves for a model in population dynamics with nonlocal consumption of resources, Math. Model. Nat. Phenom., $\mathbf{1}, \mathbf{1}$ (2006), 63-80

[9] P.D Hislop, I.M. Sigal. Introduction to spectral theory. With applications to Schrödinger operators. Springer, 1996, 337 pp.

[10] J.-L. Lions, E. Magenes. Problèmes aux limites non homogènes et applications. Vol. 1. Dunod, Paris, 1968, 372 pp.

[11] P. Manandhar, J. Jang, G.C. Schatz, M.A. Ratner, S. Hong. Anomalous surface diffusion in nanoscale direct deposition processes, Phys. Rev. Lett., 90 (2003), 4043-4052 
[12] R. Metzler, J. Klafter. The random walk's guide to anomalous diffusion: a fractional dynamics approach, Phys. Rep., 339 (2000), 1-77

[13] J. Sancho, A. Lacasta, K. Lindenberg, I. Sokolov, A. Romero. Diffusion on a solid surface: Anomalous is normal, Phys. Rev. Lett., 92 (2004), 250601

[14] H. Scher, E. Montroll. Anomalous transit-time dispersion in amorphous solids, Phys. Rev. B, 12 (1975), 2455-2477

[15] T. Solomon, E. Weeks, H. Swinney. Observation of anomalous diffusion and Levy flights in a two-dimensional rotating flow, Phys. Rev. Lett., 71 (1993), 3975-3978

[16] L.R. Volevich. Solubility of boundary value problems for general elliptic systems, Mat. Sb., 68, 110 (1965), 373-416; English translation: Amer. Math. Soc. Transl., 67, 2 (1968), 182-225.

[17] V. Volpert. Elliptic partial differential equations. Volume I. Fredholm theory of elliptic problems in unbounded domains. Birkhäuser, 2011, 639 pp.

[18] V. Volpert, B. Kazmierczak, M. Massot, Z.Peradzynski. Solvability conditions for elliptic problems with non-Fredholm operators, Appl. Math., 29, 2 (2002), 219-238

[19] V. Vougalter, V. Volpert. Solvability conditions for some non Fredholm operators, Proc. Edinb. Math. Soc. (2), 54, 1 (2011), 249-271

[20] V. Vougalter, V. Volpert. On the solvability conditions for some non Fredholm operators, Int. J. Pure Appl. Math., 60, 2 (2010), 169-191

[21] V. Vougalter, V. Volpert. On the solvability conditions for the diffusion equation with convection terms, Commun. Pure Appl. Anal., 11, 1 (2012), 365-373

[22] V. Vougalter, V. Volpert. Solvability relations for some non Fredholm operators, Int. Electron. J. Pure Appl.Math., 2, 1 (2010), 75-83

[23] V. Volpert, V. Vougalter. On the solvability conditions for a linearized CahnHilliard equation, Rend. Istit. Mat. Univ. Trieste, 43 (2011), 1-9

[24] V. Vougalter, V. Volpert. Solvability conditions for some systems with non Fredholm operators, Int. Electron. J. Pure Appl.Math., 2, 3 (2010), 183-187

[25] V. Vougalter, V. Volpert. Solvability conditions for some linear and nonlinear non-Fredholm elliptic problems, Anal. Math. Phys., 2, 4 (2012), 473-496 